\title{
"I enjoy sex more without condoms": revisiting barriers and facilitators of consistent condom use among adolescents and young adults in South Africa
}

Anthony Ajayi ( $\nabla$ ajayianthony@gmail.com )

University of Fort Hare East London Campus https://orcid.org/0000-0002-6004-3972

Olumuyiwa Omonaiye

Deakin University

Charlotte Nwogwugwu

University of Maryland Baltimore

\section{Research}

Keywords: Condoms, consistent condom use, partner communication, adolescents, young adults, selfefficacy, HIV

Posted Date: March 15th, 2021

DOI: https://doi.org/10.21203/rs.3.rs-271620/v2

License: (c) (1) This work is licensed under a Creative Commons Attribution 4.0 International License.

Read Full License 


\section{Abstract}

Background Previous studies have examined consistent condom use correlates in South Africa, focusing on sociodemographic factors, HIV risk perceptions, relationship conflict, multiple sexual partners, and masculinity. However, the effect of family financial support, HIV testing, partner communication and selfefficacy for HIV prevention is less studied. We drew from a cross-sectional survey to address this gap and highlight the key barriers and facilitators of consistent condom use among young people. Methods We analysed data obtained from 631 unmarried sexually active male and females students selected using stratified sampling from a university in Eastern Cape Province of South Africa. Consistent condom use was defined as regular use of condoms in all sexual encounters in the past year. We used an open-ended question to probe the reasons for inconsistent condom use. Adjusted and unadjusted regression analysis were fitted to examine factors associated with consistent condom use. Results The prevalence of consistent condom use was 39.3\% (Cl: 35.5\%-43.2\%), with no significant gender and age differences. After adjusting for relevant covariates, living with foster parents (AOR; $1.8095 \% \mathrm{Cl}$; 1.09-2.97), adequate family financial support (AOR; $2.4995 \% \mathrm{Cl}$; 1.71-3.62), partner knew status (AOR; $1.9195 \% \mathrm{Cl} ; 1.09-3.37$ ) and feeling confident in one's ability to prevent HIV (AOR; $1.7795 \% \mathrm{Cl} ; 1.09-2.86)$ were associated with increased odds of consistent condom use. However, self-report of low condom self-efficacy (AOR; 0.58 $95 \% \mathrm{Cl} ; 0.40-0.85$ ) and alcohol use (AOR; $0.8395 \% \mathrm{Cl}$; 0.58-1.19) were associated with lower odds of consistent condom use. Young people who inconsistently used condoms reiterated that sex is often unplanned and condoms are not always available. The desire for maximum pleasure, partner's objection, trust, and use of hormonal contraceptives were further reasons others inconsistently use condoms. Conclusions Inconsistent condom use remains a challenge among unmarried sexually active young people in South Africa. Education of young people on the need for partner communication about HIV, HIV testing uptake, and condom use should happen more rigorously in school and through the media to improve their consistent use of condoms.

\section{Introduction}

South Africa remains the epicentre of the HIV pandemic, with 7.7 million people living with HIV and 240,000 new infections every year [1]. Horizontal transmission remains the predominant route for HIV transmission in the country, particularly among young people [2]. Compelling scientific evidence has shown that the correct and consistent use of condoms during sexual intercourse represents one of the most viable means of preventing HIV and sexually transmitted infections (STIs) [3, 4]. In South Africa, condoms are widely available at no cost to young adults through several outlets, such as youth centres and public health facilities [5]. An estimated $87 \%$ of young adults in the country reported that it would be effortless to obtain condoms [5].

Despite the availability and effectiveness of condoms in preventing HIV, STIs and unwanted pregnancies, many unmarried adolescents and young adults in South Africa are not using condoms at all or inconsistently use condoms [6, 7]. According to the 2017 South African National HIV Prevalence, Incidence, Behaviour, and Communication Survey, $68 \%$ of young male adults with multiple partners 
reported using condoms the last time they had sex, compared to $47 \%$ of young female adults [8]. Not surprisingly, in 2018, new HIV infections among young female adults aged 15-24 years were more than double those among young male adults: 69,000 new infections among young female adult women, compared to 25,000 among young male adults [1]. However, complex structural, economic, and social factors, make young women more vulnerable to HIV acquisition, including poverty, gender norms, and sexual violence, among others [9].

Indeed, condoms have hardly ever been considered as being attractive or convenient to attain sexual pleasure; neither has it been linked as a vehicle for transmission of trust between sexual partners [10]. Trust based on sexual intimacy has been linked with unprotected sexual intercourse and inconsistent condom use with increased risk of HIV and STIs [11]. Hence, the positive feeling that has been cultivated between sexual partners and duration of sexual relationship plays a significant role in determining the perceived need for safe sexual practices, which include the use of condom [10,12]. Consequently, partner communication about HIV risk reduction regardless of trust is crucial for safer sex practices, including consistent use of condoms. Communication about HIV with sexual partners, such as talking about condom use or HIV testing, can serve as a foundation for behavioural change [13]. Partner communication has been linked to increased HIV testing and condom use [13].

Also, condom fatigue, low perceived personal risk of sexually transmitted diseases, and low condom selfefficacy are some of the well-documented reasons for inconsistent condom use [14-17]. Specifically, in South Africa, low condom use self-efficacy has been associated with inconsistent condom use among young adults [18], and low self-efficacy is linked with increased unprotected sexual activities [19]. As a theoretical construct, self-efficacy is part of Bandura's social cognitive theory and refers to an individual's belief in his or her capability to perform behaviours essential to produce specific performance attainments [20]. Condom use self-efficacy refers to an individual's self-belief in his/her ability to obtain condoms, negotiate the use of condoms with partners, and use condoms during penetrative sexual acts [21-23]. Thus, individuals who have a high sense of condom self-efficacy are likely to use condoms, while individuals who have reservations about their ability to use condoms are less likely to do so [14]. Evidence suggests that counselling South African young adults on condom self-efficacy can lead to the promotion and use of condoms with attendant HIV risk reduction [24].

Previous studies have examined consistent condom use correlates in South Africa, focusing on sociodemographic factors, HIV risk perceptions, relationship conflict, multiple sexual partners, and masculinity. However, gaps remain in our understanding of barriers and facilitators of consistent condom use among young people. Specifically, our understanding of the role of partner communication and HIV prevention self-efficacy remains limited. While a previous study suggests that HIV risk perceptions is not significantly associated with consistent condom use[25], expression of self-efficacy for HIV prevention could influence consistent condom use. Also, partner communication could facilitate regular use of condoms; however, the link between partner communication about HIV/STIs and consistent condom use has only been sparsely explored. Also, previous studies have mostly focused on prevalence and correlates of consistent use without probing the reasons for inconsistent condom use. This is a miss 
opportunity given that asking an open-ended question probing reasons for inconsistent use of condoms among a large sample of non and inconsistent users could produce new insights to enhance our understanding of barriers to consistent condom use. Our study addresses this gap by examining barriers and facilitators of consistent condom use among sexually active adolescents and young adults aged 1724 years. Our findings could be critical in developing HIV prevention strategies and programs to mitigate the spread of HIV among young people in South Africa.

\section{Methods}

We analysed the data from the adolescents and young women's sexual health survey conducted among students of a tertiary institution in Eastern Cape, South Africa between June and November 2018. The study was conducted among adolescents and young women aged 16-24, given the heightened risk of HIV acquisition in this cohort [1]. Despite the significant progress in reducing new HIV infection, young people, especially girls, remain disproportionately at risk of HIV infection [1]. Students were chosen for this study conveniently due to the paucity of funds for a household survey. We conducted the study in a government-owned university in Eastern Cape Province, South Africa. Students were included if they were within the age of 16 and 24 years and a university student at selected campus. Students visiting from other universities were ineligible for this study. Respondents were selected using stratified sampling. Gender, year of study, faculty of study were the stratification unit. We selected a proportionate sample size per the strata's population (See Table 1). More females were included in the study, given that the ratio of male to female students is 2:3. The full details of the study methodology have been presented elsewhere [26, 27].

Table 1: Overview of sample selection

\begin{tabular}{|lll|}
\hline Faculties & Total participants & Number selected in each year of study \\
\hline Education & 246 & 82 \\
\hline Health sciences & 100 & 25 \\
\hline Management and commerce & 150 & 50 \\
\hline Law & 120 & 30 \\
\hline Social Science and Humanities & 217 & 72 \\
\hline Total & 833 & \\
\hline
\end{tabular}

Overall, 833 respondents took part in the main study. However, only 631 respondents who were not married but sexually active in the past one year were included in the study. For this study, a sample size of 631 was deemed appropriate at $\pm 3.96 \%$ precision level, a $95 \%$ confidence level, $39 \%$ prevalence of consistent condom use [25] and a population of 15,000, adjusted for $10 \%$ possible attrition. Well trained research assistants who were graduate students administered the study instrument using Open Data Kits 
(ODK) application installed on android device. The use of ODK granted the respondents privacy to complete the study questionnaire and without distraction from and interference of friends. Participants were recruited over six months in their lecture rooms. They were approached and invited to participate in the study and those who consented completed the instrument either on their android enable phones or on the research assistants' phone in private spaces earmarked for the study. The response rate was high, at $89 \%$.

\section{Ethical considerations}

The ethical review committee of the University of Fort Hare approved the study protocol. All Institutional Review Board (IRB) guidelines for conducting human subject research were followed throughout the study. Participation was voluntary, and respondents provided written consent indicating they understood the study's purpose and the use of data obtained. Respondents reserved the right not to answer any questions they were uncomfortable with and to withdraw their participation from the study altogether. The right of respondents to privacy, confidentiality, and anonymity was protected throughout the study. No personal identifying information was collected.

\section{Measures}

This study's main outcome was consistent condom use, defined as the use of condoms at every sexual encounter over the past year. We asked sexually active respondents if they used condoms regularly at every sexual intercourse in the past year, and dichotomised their responses as "yes" or "no". We probed for reasons for not consistently using condoms with an open-ended question. Responses were coded and grouped under six main themes.

\section{Independent variables}

We included three main independent variables-partner communication, HIV prevention and condoms self-efficacy - in this study. To measure partner communications, we asked respondents if they had discussed HIV/STIs with their partners, knew their partner's HIV status, and if their partner knew their serostatus. Responses to these questions were coded as yes or no. HIV prevention self-efficacy was measured by asking participants if they feel confident about their ability to prevent themselves from contracting HIV. Condom self-efficacy was measured with 13 items rated on a 3-point Likert scale with categories 1 (agree), 2 (Neutral) and 3 (Agree). This self-rated construct has been previously validated by Barkley and colleagues [28]. The construct includes questions like "I would feel confident in my ability to put a condom on myself or my partner, purchase condoms, carry condoms, remove condoms after sex, refuse to have sex with someone not willing to use condoms and insist on condom use every time I have sex. We assess the construct's internal consistency and reliability in our study setting, and the analysis yielded a Cronbach alpha reliability estimate of 0.81 . Responses to reverse questions were recoded such that all questions align in one direction with lower scores indicating high condom self-efficacy and higher score indicating low self-efficacy. Thirty-nine scores were obtainable, and 1 to 19 was categorised as higher condom self-efficacy while 20 and above were categorised as low self-efficacy. 


\section{Covariates}

We included three main categories of covariates, including demographic factors (age and sex), family factors (family structure and family support) and behavioural factors (HIV testing and alcohol use). Age was measured as a continuous variable but later categorised as $16-19$ years and 20-24 years. Family structure was coded as single-parent family, two-parents family and foster family. Family financial support is defined as the perceived rating of support received from parents. This was a substitute for the measure of parental socioeconomic status. Respondents rated the financial support they got from their parents as adequate, moderate, insufficient, no support. We only included the current use of alcohol in this study by categorising respondents into current users and non-users. Current users are those who drank alcohol in the past year. Lastly, we measured HIV testing by asking if respondents had ever tested for HIV or not. Lastly, we categorised respondents into six categories based on their sexual behaviour in the past year. These categories include one steady partner only, two steady partners, three or more steady partners, one casual partner only, concurrent main and casual partner, multiple concurrent partners.

\section{Data analysis}

We used Stata version 15 to analyse the dataset. We performed descriptive statistics for all variables of interests. Specifically, we computed mean and standard deviation for age and frequency counts and percentages for all categorical variables. All analyses were stratified by gender. Also, we used adjusted and unadjusted logistics regression to examine the effect of partner communication, HIV prevention selfefficacy and condom self-efficacy on consistent condom use. We stratified the adjusted and unadjusted regression analyses by gender to examine the pattern of the effect of partner communication, HIV prevention self-efficacy and condom self-efficacy on consistent condom use among males and females. The analysis was performed at $95 \%$ confidence level, and $p$-values $<0.05$ were considered statistically significant. We coded responses to the open-ended questions on reasons for inconsistent condom use. Codes were reviewed by the research team and similar codes were merged under a common theme. We discussed each theme and used verbatim quotes to support out descriptions. Also, we used simple frequency to indicate how frequent participants mentioned them.

\section{Results}

\section{Descriptive findings}

Participants mean age was 21.23 years (SD \pm 1.70 ). As presented in Table 2, most respondents were aged 20-24 years (82.6\%), received inadequate family financial support (63.7\%), have ever tested for HIV (70.2\%), felt confident in their ability to prevent themselves from contracting HIV/STIs (71.8\%). Less than half of the respondents were from a single-parent family (44.9\%). More females (76.4\%) had tested for HIV compared to males (60.4\%). Over half of the respondents were current alcohol users (56.3\%), including $46.4 \%$ of females and $52.1 \%$ of males. Slightly over half $(52.8 \%)$ of the respondents had ever discussed HIV/STIs with their partners. However, partner communication about HIV/STIs is higher among more females (55.4\%) than males (48.6\%). Only two-fifth of both male and female respondents knew 
their partner's HIV status. However, close to half of the respondents (48.0\%) affirmed that their partner knew their status. A higher proportion of females (52.9\%) affirmed that their partner knew their status than males (40.4\%). Lastly, about half of the male and female respondents had a high condom selfefficacy score.

Table 2: Demographic, family and behavioural characteristics of respondents stratified by gender 


\begin{tabular}{|c|c|c|c|}
\hline Variables & $\begin{array}{l}\text { Female } \\
n=386\end{array}$ & $\begin{array}{l}\text { Male } \\
n=245\end{array}$ & $\begin{array}{l}\text { All respondents } \\
n=631\end{array}$ \\
\hline \multicolumn{4}{|l|}{ Age } \\
\hline $16-19$ & $68(17.6)$ & $42(17.1)$ & $110(17.4)$ \\
\hline $20-24$ & $318(82.4)$ & $203(82.9)$ & $521(82.6)$ \\
\hline \multicolumn{4}{|l|}{ Family structure } \\
\hline Single parent family & $174(45.1)$ & $109(44.5)$ & $283(44.9)$ \\
\hline Both parents family & $144(37.3)$ & $99(40.4)$ & $243(38.5)$ \\
\hline Foster parent family & $68(17.6)$ & $37(15.1)$ & $105(16.6)$ \\
\hline \multicolumn{4}{|l|}{ Family financial support } \\
\hline Adequate & $132(34.2)$ & $97(39.6)$ & $229(36.3)$ \\
\hline Inadequate & $254(65.8)$ & $148(60.4)$ & $402(63.7)$ \\
\hline \multicolumn{4}{|l|}{ Ever tested for HIV/STIs } \\
\hline Yes & $295(76.4)$ & $148(60.4)$ & $443(70.2)$ \\
\hline No & $91(23.6)$ & $97(39.6)$ & $188(29.8)$ \\
\hline \multicolumn{4}{|l|}{ Discussed HIV/STIs with partners } \\
\hline Yes & $214(55.4)$ & $119(48.6)$ & $333(52.8)$ \\
\hline No & $172(44.6)$ & $126(51.4)$ & $298(47.2)$ \\
\hline \multicolumn{4}{|l|}{ Know partner status } \\
\hline Yes & $156(40.4)$ & $98(40.0)$ & $254(40.3)$ \\
\hline No & $230(59.6)$ & $147(60.0)$ & $377(59.8)$ \\
\hline \multicolumn{4}{|l|}{ Partner know status } \\
\hline Yes & $204(52.9)$ & $99(40.4)$ & $303(48.0)$ \\
\hline No & $182(47.2)$ & $146(59.6)$ & $328(52.0)$ \\
\hline \multicolumn{4}{|c|}{ Feel confident in my ability to prevent HIV } \\
\hline Yes & $284(73.6)$ & $169(69.0)$ & $453(71.8)$ \\
\hline No & $102(26.4)$ & $76(31.0)$ & $178(28.2)$ \\
\hline \multicolumn{4}{|l|}{ Condom self-efficacy } \\
\hline High self-efficacy score $\geq 20$ & $190(49.2)$ & $122(49.8)$ & $312(49.5)$ \\
\hline
\end{tabular}




\begin{tabular}{|llll|}
\hline Low self-efficacy score $<20$ & $196(50.8)$ & $123(50.2)$ & $319(50.6)$ \\
\hline Yes & & & \\
No & $201(52.1)$ & $138(56.3)$ & $292(46.3)$ \\
\hline Sexual behaviours in the past year & $185(47.9)$ & $107(56.3)$ & $339(53.7)$ \\
\hline Only one steady partner & & & \\
\hline Two steady but not concurrent partners & $206(53.4)$ & $71(29.0)$ & $277(43.9)$ \\
\hline Three or more but not partners & $70(18.1)$ & $39(15.9)$ & $109(17.3)$ \\
\hline Only one casual partner & $35(9.1)$ & $36(14.7)$ & $71(11.3)$ \\
\hline Both main partner and casual partner concurrently & $35(9.1)$ & $23(9.4)$ & $58(9.2)$ \\
\hline Multiple concurrent partners & $27(7.0)$ & $61(24.9)$ & $88(14.0)$ \\
\hline
\end{tabular}

The prevalence of consistent condom use was 39.3\% (95\% Cl: $35.5 \%-43.2 \%)$. There was no statistical difference in the proportion of male (39.2\% Cl: $33.0 \%-45.6 \%)$ and female (39.4\% Cl:34.5\%-44.4\%) consistent condom users. As shown in Table 3, there was no significant age difference in condom use consistency proportion. However, consistent condom use prevalence was higher among respondents from a foster family $(45.7 \%)$ relative to single-parent families $(36.8 \%)$. This difference is even more pronounced in the stratified analysis for females, where over half $(52.9 \%)$ of those from a foster family reported using condoms consistently compared to only $36.2 \%$ of those from a single-parent family. Even though a higher proportion of males from a two-parent family (43.4\%) than those from a foster family (32.4\%) reported consistent condom use, the difference was not statistically significant. Respondents who received adequate family financial support (53.3\%) reported a higher rate of consistent condom use than those who received inadequate financial support (31.3\%). A similar pattern was observed among males and females in the stratified analysis. Individuals who had tested for HIV (44.0\%) reported a higher rate of consistent condom use than those who had never tested for HIV (28.2\%). The pattern holds among males and females in the stratified analysis. The prevalence of consistent condom use was lower among current alcohol users (34.5\%) than non-users (44.9\%), and this result was consistent for both male and females in the stratified analysis.

Young people who had discussed HIV/STIs with their partners (42.0\%) tend to use condoms more consistently than those who never did (36.2\%). This is especially true among males, where $46.2 \%$ of those who had discussed HIV/STIs with their partners reported consistent condom use compared to only $32.5 \%$ of those who never did. There was, however, no difference in the proportion of consistent condom users by the discussion of HIV/STIs with sexual partner among females. A higher proportion of those who knew their partner's status $(42.1 \%)$ than those who did not $(37.4 \%)$ reported using condoms consistently, but the difference was not statistically significant. 
Similarly, those whose partner knew their status (45.2\%) were significantly more likely to use condoms than those whose partner was unaware of their status (33.8\%). Over half of males whose partner knew their status used condoms consistently compared to only $31.5 \%$ of those whose partners were unaware of their status. Whereas, the rate was slightly lower among females with $42.7 \%$ of those whose partner knew their HIV status, reporting consistent condom use compared to $35.7 \%$ of those whose partners were unaware. Young people who reported having self-efficacy for HIV prevention (45.3\%) were significantly more likely to use condom consistently than those who reported no confidence in their ability to prevent HIV (24.2\%). The pattern persists among males and females. Also, young people with high condom selfefficacy (49.4\%) were significantly more likely to use condoms consistently than those with low condom self-efficacy (29.5\%). This was true among males as well as females in the stratified analysis.

Table 3: Pearson Chi-Square statistical analysis showing association between partner communication, condom self-efficacy, demographic, family and behavioural factors and consistent condom use stratified by gender 


\begin{tabular}{|c|c|c|c|}
\hline Variables & All & Female & Male \\
\hline All & 248 (39.3) & $152(39.4)$ & 96 (39.2) \\
\hline \multicolumn{4}{|l|}{ Age } \\
\hline $16-19$ & $45(40.9)$ & $26(38.2)$ & $77(37.9)$ \\
\hline $20-24$ & $203(39.0)$ & $126(39.6)$ & $19(45.2)$ \\
\hline \multicolumn{4}{|l|}{ Family structure } \\
\hline Single parent family & $104(36.8)$ & $63(36.2)$ & $41(37.6)$ \\
\hline Both parents family & $96(39.5)$ & $53(36.8)$ & $43(43.4)$ \\
\hline Foster parent family & 48 (45.7) & $36(52.9)^{\star}$ & $12(32.4)$ \\
\hline \multicolumn{4}{|l|}{ Family support } \\
\hline Adequate & 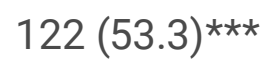 & $67(50.8) \star$ & $55(56.7)^{\star \star \star}$ \\
\hline Inadequate & $126(31.3)$ & $85(33.5)$ & $41(27.7)$ \\
\hline \multicolumn{4}{|l|}{ Ever tested for HIV } \\
\hline Yes & 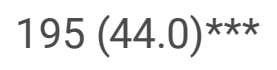 & $126(42.7)^{\star}$ & $69(46.6)^{*}$ \\
\hline No & $53(28.2)$ & $26(28.6)$ & $27(27.8)$ \\
\hline \multicolumn{4}{|l|}{ Discussed HIV/STIs with partners } \\
\hline Yes & $140(42.0)$ & $85(39.7)$ & $55(46.2)^{*}$ \\
\hline No & $108(36.2)$ & $67(39.0)$ & $41(32.5)$ \\
\hline \multicolumn{4}{|l|}{ Know partner status } \\
\hline Yes & $107(42.1)$ & $66(42.3)$ & $41(41.8)$ \\
\hline No & $141(37.4)$ & $86(37.4)$ & $55(37.4)$ \\
\hline \multicolumn{4}{|l|}{ Partner know status } \\
\hline Yes & $137(45.2)^{\star}$ & $87(42.7)$ & $50(50.5)^{\star}$ \\
\hline No & $111(33.8)$ & $65(35.7)$ & $46(31.5)$ \\
\hline \multicolumn{4}{|c|}{ Feel confident in my ability to prevent HIV } \\
\hline Yes & 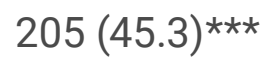 & $124(43.7)^{\star}$ & $81(47.9)^{\star \star \star}$ \\
\hline No & $43(24.2)$ & $28(27.5)$ & $15(19.7)$ \\
\hline \multicolumn{4}{|l|}{ Condom self-efficacy } \\
\hline High self-efficacy score $\geq 20$ & $154(49.4)^{\star \star \star}$ & $92(48.4)^{\star \star \star}$ & $62(50.8)^{\star \star \star}$ \\
\hline
\end{tabular}




\begin{tabular}{|llll|}
\hline Low self-efficacy score $>20$ & $94(29.5)$ & $60(30.6)$ & $34(27.6)$ \\
\hline Yes & & & \\
\hline No & $117(34.5)^{\star}$ & $71(35.3)$ & $46(33.3)$ \\
\hline Sexual behaviours in the past year & $131(44.9)$ & $81(43.8)$ & $50(46.7)$ \\
\hline Only one steady partner & & & \\
\hline Two steady but not concurrent partners & $277(43.9)$ & $206(53.4)$ & $71(29.0)$ \\
\hline Three or more but not partners & $109(17.3)$ & $70(18.1)$ & $39(15.9)$ \\
\hline Only one casual partner & $71(11.3)$ & $35(9.1)$ & $36(14.7)$ \\
\hline Both main partner and casual partner concurrently & $58(9.2)$ & $35(9.1)$ & $23(9.4)$ \\
\hline Multiple concurrent partners & $88(14.0)$ & $27(7.0)$ & $61(24.9)$ \\
\hline
\end{tabular}

\section{Multivariable findings}

The results of the adjusted and unadjusted logistic regression are presented in Tables 4. In the unadjusted model, age, family structure and gender were not significantly associated with consistent condom use. This pattern persists in the adjusted model.

Alcohol use was associated with lower odds of consistent condom use in the unadjusted model. The direction and magnitude of the association persist in the adjusted model. Also, adequate family financial support was associated with higher odds of consistent condom use in the unadjusted model. The direction and magnitude of effect remained in the adjusted model, indicating that adequate family financial support could facilitate consistent condom use. Similarly, having tested for HIV was associated with a higher likelihood of consistent condom use in the unadjusted regression model. However, while the direction of effect persists after adjusting for relevant covariates, the magnitude of effect reduced significantly, and the confidence interval crosses one.

On partner communication, discussion of HIV/STIs with partners was not significantly associated with a higher likelihood of consistent condom use in both unadjusted and adjusted models. Similarly, knowing one's partner's status was not significantly associated with consistent condom use for all respondents in both the unadjusted and adjusted models. However, revealing one's HIV status to one's partner was significantly associated with higher odds of consistent condom use. The magnitude and direction of effect remained in the adjusted model, providing clear and robust evidence of the association of informing partner of one's HIV status with higher odds of consistent condom use. 
The unadjusted model also shows that self-efficacy for HIV prevention was associated with a higher probability of consistent condom use. The direction and magnitude of effect persist in the adjusted model, suggesting that self-efficacy for HIV prevention was significantly associated with a higher likelihood of consistent condom use. Similarly, low self-efficacy for condom use was associated with lower odds of consistent condom use for all in the unadjusted model. The direction and magnitude of effect persist in the adjusted model for all participants.

Table 4: Unadjusted logistic regression model 


\begin{tabular}{|c|c|c|}
\hline \multirow[t]{2}{*}{ Variables } & \multicolumn{2}{|l|}{ All participants } \\
\hline & Unadjusted odds $(\mathrm{Cl})$ & adjusted odds $(\mathrm{Cl})$ \\
\hline \multicolumn{3}{|l|}{ Gender } \\
\hline Male & $0.99(0.71-1.38)$ & 1. $(0.88-1.90)$ \\
\hline Female & 1 & 1 \\
\hline \multicolumn{3}{|l|}{ Age } \\
\hline $16-19$ & $1.08(0.71-1.65)$ & $1.04(0.65-1.65)$ \\
\hline $20-24$ & 1 & 1 \\
\hline \multicolumn{3}{|l|}{ Family structure } \\
\hline Single parent family & 1 & 1 \\
\hline Both parents family & $1.12(0.79-1.60)$ & $1.08(0.73-1.59)$ \\
\hline Foster parent family & $1.45(0.92-2.28)$ & $1.80(1.09-2.97)^{\star}$ \\
\hline \multicolumn{3}{|c|}{ Family financial support } \\
\hline Adequate & $2.50(1.79-3.49)^{\star \star \star}$ & $2.49(1.71-3.62)^{\star \star \star}$ \\
\hline Inadequate & 1 & 1 \\
\hline \multicolumn{3}{|l|}{ Current alcohol users } \\
\hline Yes & $0.65(0.47-0.89) *$ & $0.83(0.58-1.19)$ \\
\hline No & 1 & 1 \\
\hline \multicolumn{3}{|l|}{ Ever tested for HIV } \\
\hline Yes & $2.00(1.38-2.90)^{\star \star \star}$ & $1.13(0.67-1.88)$ \\
\hline No & 1 & 1 \\
\hline \multicolumn{3}{|c|}{ Discussed HIV/STIs with partners } \\
\hline Yes & $1.28(0.93-1.76)$ & $0.64(0.39-1.05)$ \\
\hline No & 1 & 1 \\
\hline \multicolumn{3}{|l|}{ Know partner status } \\
\hline Yes & $1.22(0.88-1.69)$ & $0.67(0.42-1.09)$ \\
\hline No & 1 & 1 \\
\hline \multicolumn{3}{|l|}{ Partner know status } \\
\hline Yes & $1.61(1.17-2.23)^{\star}$ & $1.91(1.09-3.37)^{\star}$ \\
\hline
\end{tabular}


Feel confident in one's ability to prevent HIV/STIs

\begin{tabular}{|lll|}
\hline Yes & $2.60(1.76-3.83)^{\star \star \star}$ & $1.77(1.09-2.87)^{\star}$ \\
\hline No & 1 & 1 \\
\hline Condom self-efficacy & & \\
\hline High self-efficacy score $\geq 20$ & 1 & 1 \\
\hline Low self-efficacy score $>20$ & $0.43(0.31-0.59)^{\star \star \star}$ & $0.58(0.40-0.85)^{\star}$ \\
\hline Sexual behaviours in the past year & & \\
\hline Only one steady partner & 1 & 1 \\
\hline Two steady but not concurrent partners & $0.70(0.44-1.10)$ & $0.79(0.48-1.29)$ \\
\hline Three or more but not partners & $0.53(0.31-0.93)^{\star}$ & $0.60(0.32-1.12)$ \\
\hline Only one casual partner & $1.72(0.78-3.81)$ & $2.27(0.96-5.38)$ \\
\hline Both main partner and casual partner concurrently & $0.63(0.35-1.14)$ & $0.95(0.50-1.79)$ \\
\hline Multiple concurrent partners & $0.21(0.11-0.39)^{\star \star \star}$ & $0.24(0.12-0.48)^{\star \star \star}$ \\
\hline
\end{tabular}

\section{Reasons for inconsistent condom use}

To understand why young people did not use condoms consistently or not use it all in a high HIV prevalent setting like South Africa, we asked those not using condoms consistently to provide open-ended answers to explain their reasons. Six main reasons emerged from the content analysis of reasons for inconsistent condom use. Of the 185 respondents who provided reasons for their inconsistent condom use, the desire for maximum pleasure was the main concerns of $14.6 \%$ of them, while $16.8 \%$ said they just liked to have sex without condoms (Table 5). This group of non-users believed that condoms reduce or hinder their ability to derive maximum pleasure from sex. Lack of availability of condoms was the main reason for inconsistent condom use for about one-third of non-users. They explained that sex is often unplanned and sometimes happens so fast that they often forget to use condoms. Also, unplanned sex means condoms are unavailable and sex could not wait until they are able to access condoms.

Also, trust was among the main reason for inconsistent condom use for about one-fifth of those who did not use condoms consistently. HIV testing played no role in trusting their partner, rather engaging in committed relationship and fidelity to and perceived fidelity of their partner motivated them to engage in condomless sex. Only a few of them had tested for HIV and considered themselves to carry no risk of contracting HIV from their partner.

Another one-tenth did not use condoms consistently because they relied on hormonal contraceptives. Preventing pregnancy was the primary concern of this group of inconsistent condom users and hormonal 
contraceptives help them realise this goal. They appear to be more concerned about preventing pregnancy and less concerned about preventing HIV/STIs. A few of them $(7.0 \%)$ however blamed their partner for refusing to use condoms.

Table 5: Reasons for inconsistent condom use

\begin{tabular}{|llc|}
\hline Reasons for inconsistent condom use & Frequency $(n=185)$ & Percent \\
\hline Condoms unavailable, sex was not planned, happened so fast & 58 & 31.4 \\
\hline We don't feel like it & 31 & 16.8 \\
\hline Trust partner & 35 & 18.9 \\
\hline Pleasure concerns & 27 & 14.6 \\
\hline On hormonal contraceptives & 21 & 11.4 \\
\hline Partner objects & 13 & 7.0 \\
\hline
\end{tabular}

\section{Discussion}

In a high HIV prevalent setting, consistent condom use promotion is one of the tools to combat the spread of HIV, especially among adolescent and young adults (aged 15-24) known to have the highest rate of new infections. Our study examined the prevalence, barriers and facilitators of consistent condom use among sexually active adolescents and young adults. We found that only two-fifths of our study participants consistently used condoms in the past year, which is in line with previous studies [29,30] among young people in South Africa. However, a study conducted reported a lower prevalence among young women in rural settings [31]. In contrast, a study among recently circumcised males and another conducted among young people in Cape Town reported a slightly higher consistent condom use prevalence [6,32].

Surprisingly, this study's finding is similar to previous studies in other settings with a relatively low HIV prevalence [25,33] compared with South Africa. Even though the need to prevent STIs and unintended pregnancy is universal, we expected that the prevalence of consistent condoms use among young people would be higher in South Africa than those reported among a similar population [25] in a low prevalent setting, like Nigeria. Contrary to a previous study in South Africa[30], we did not observe any gender differences in condom use consistency. Also, the factors associated with consistent condom use are similar for both males and females, underscoring the absence of differences in condom behaviours of young men and women.

This study highlighted factors that facilitate consistent condom use, some of which have been reported in previous studies [25, 30-32]. We found that family financial support, disclosure of status to partner, HIV testing, and self-efficacy to prevent HIV status positively impact consistent condom use. While the influence and importance of condom self-efficacy are well established in the literature, our study 
highlights the considerable importance of family financial support, partner communication, HIV testing uptake and self-efficacy to prevent HIV/STIs. Previous studies have not explored the link between family financial support and condom use consistency. Our plausible explanation for why young people who received adequate financial support use condoms more consistently than those who did not is that they may have sufficient resources to purchase condoms than their counterparts. They could, for instance, purchase and keep their preferred condoms brands in their rooms in anticipation of sex, unlike their counterparts who may have to ration their scarce resources and advance purchase of condoms may not be among their priorities. It is worth noting that condoms are distributed on campus, but this happens infrequently and may not go far enough in solving condoms unavailability problem.

Previous studies have highlighted the importance of partner communication on protective sexual behaviours $[25,34]$. We know from these studies that partners who discussed the need to use condoms and prevent unintended pregnancy are more likely to engage in protective sex. Our study further adds to the emerging evidence of the importance of partner communication for HIV/STIs and unintended pregnancy prevention. Partner communication on HIV/STIs means that couples would risk and resort to the appropriate preventive methods.

Consistent with the literature [25,30], our study shows that HIV testing encourages protective behaviours. Those who have tested are more likely to use condoms consistently than those who never tested for HIV. The difference observed could be due to the positive impact of the counselling before and after HIV testing. Also, uptake of HIV testing is a positive health behaviour, suggesting that most people who undertook HIV testing are more concerned about preventing HIV than those who had never been tested.

However, no studies have examined the influence of HIV prevention self-efficacy. Expression of high selfefficacy for HIV prevention reflects that young people are not only aware of methods of preventing HIV/STIs but also feel confident in their ability to protect themselves. Therefore, it is not surprising to see that those who expressed confidence in their ability to prevent HIV/STIs are more likely to use condoms consistently.

Based on our findings, the desire for maximum pleasure, preference for sex without condoms, unavailability of condoms, partners' objections, alcohol use, and hormonal contraception use constitute barriers to consistent condom use. Like previous studies, our study demonstrates that young people who believe that condoms reduce sex pleasure are unlikely to use it regularly, if at all. It appears that the desire for maximum fun trumps the need to prevent HIV/STIs for many young people. Also, in line with previous studies, condom unavailability was reported as part of the factors hindering condom use consistency [25, 30]. For many, sexual intercourse is unplanned, and in such situations, condoms are not always available. Rather than wait to source for condoms, some young people often take the risk, ignoring the possibility of contracting HIV/STIS.

Trust has also been documented in the literature as one of the barriers to condom use consistency among young people. In this study, young people failed to use condoms consistently because they trust their partners to remain faithful in the relationship. The trust is based on perceived fidelity in a 
relationship. Since some already tested for HIV and were on hormonal contraception, they do not see the need to use condoms. For others, the trust could be described as a "blind trust" given that it was implied despite having never tested for HIV. In these relationships, unprotected sex is equated with trust and initiating a conversation about condoms could be misconstrued as distrust [34]. Our result indicated that those who failed to use condoms at all or inconsistently because they were on hormonal contraception suggest that some young people are more concerned with pregnancy prevention than HIV/STI prevention. Our findings on the link between alcohol use and condom use consistency further add to the literature on the adverse effect of alcohol on high-risk sexual behaviours. Excessive use of alcohol could make young people inebriated, therefore, lacking agency to negotiate condom use.

In light of our study findings, there is a need to invest in multi-prongs interventions that begin with early and comprehensive sex education. This sex education should continue beyond high school and should happen in other settings outside of school to reach out of school adolescents and young adults. In addition to sexuality education, the government should continue to make condoms and other contraceptives freely available through the health department. The government initiative to make different varieties and flavours of condoms available is a positive development that could impact condom use consistency, given the complaint about pleasure concerns. Interventions need to be innovative and convenient while addressing these identified barriers to reach these adolescents and young adults. Counselling of students has historically been the standard intervention in safe sex education [36]. However, many young people appear not to use condoms despite counselling. To address this need, innovation on behavioural interventions are critical. Social marketing campaigns on HIV are necessary to target these groups, and therefore should be implemented more rigorously. Integrating interventions into the routine standard of care in South Africa is necessary to promote behaviour change.

\section{Study strengths and limitations}

This study adds to the literature, highlighting the barriers and facilitators of condom use consistency in a high HIV prevalent setting. The use of the mixed-methods approach is a strength of this study. Nevertheless, our study is not without some limitations. Our study population is not representative of adolescents and young adults in the country, given that they are more educated, limiting the generalisability of our findings. However, our findings are consistent with previous studies among young people living in rural and urban settings [29, 30,32], suggesting that our results may apply to other young people in the country. Even though we ensure privacy, anonymity and confidentiality throughout the study and with our use of ODK, we could not completely rule out social desirability bias, which could lead to over-estimation of the prevalence of consistent condom use.

\section{Conclusion}

Inconsistent condom use remains a challenge among unmarried sexually active young people in South Africa. Alcohol use, trust, spontaneous sex, use of hormonal contraception, partner's objection, pleasure concerns constitute barriers to consistent condom use. High condom self-efficacy, partner 
communication, HIV testing and self-efficacy for HIV prevention could facilitate consistent condom use. Education of young people on the need for partner communication about HIV, HIV testing uptake, and condom use should happen more rigorously in school and through the media to improve their consistent use of condoms. Also, interventions focusing on increasing self-efficacy for condom use and HIV prevention and risk reduction activities such as provisioning of condoms are crucial to enhancing condom use and plausibly tackling the HIV epidemic among young men and women in South Africa.

\section{Declarations}

\section{Ethics approval and consent to participate}

The ethical review committee of the University of Fort Hare approved the study protocol. All Institutional Review Board (IRB) guidelines for conducting human subject research were followed throughout the study. Participation was voluntary, and respondents provided written consent indicating they understood the study's purpose and the use of data obtained. Respondents reserved the right not to answer any questions they were uncomfortable with and to withdraw their participation from the study altogether. The right of respondents to privacy, confidentiality, and anonymity was protected throughout the study. No personal identifying information was collected.

\section{Consent for publication}

Not applicable

\section{Availability of data and material}

Data analysed in this paper is available upon request from the corresponding author.

\section{Competing interests}

We have no competing interests to declare.

\section{Funding}

We did not receive any funding for this study.

\section{Authors' contributions}

AIA conceptualised the study and conducted the analysis. All authors contributed to the draft, revision and approved the final version.

\section{Acknowledgements}

The authors acknowledge the study participants and the research assistants for their contributions. 


\section{References}

1. UNAIDS. South Africa:Overview. 2020 [cited 202030 August]; Available from: https://www.unaids.org/en/regionscountries/countries/southafrica.

2. Osuafor, G.N., et al., Condom use among married and cohabiting women and its implications for HIV infection in Mahikeng, South Africa. Journal of Population Research, 2018. 35(1): p. 41-65.

3. Foss, A.M., et al., Condoms and prevention of HIV. BMJ (Clinical research ed.), 2004. 329(7459): p. 185-186.

4. Holmes, K.K., R. Levine, and M. Weaver, Effectiveness of condoms in preventing sexually transmitted infections. Bulletin of the World Health Organization, 2004. 82(6): p. 454-461.

5. Hendriksen, E.S., et al., Predictors of condom use among young adults in South Africa: the Reproductive Health and HIV Research Unit National Youth Survey. American journal of public health, 2007. 97(7): p. 1241-1248.

6. Muchiri, E., C. Odimegwu, and N. De Wet, HIV risk perception and consistency in condom use among adolescents and young adults in urban Cape Town, South Africa: a cumulative risk analysis.

Southern African Journal of Infectious Diseases, 2017. 32(3): p. 105-110.

7. Chandran, T.M., et al., Predictors of condom use and refusal among the population of Free State province in South Africa. BMC Public Health, 2012. 12(1): p. 381.

8. Simbayi, L., et al., South African National HIV Prevalence, Incidence, Behaviour and Communication Survey, 2017. 2019, HSRC Press: Cape Town.

9. Rodrigo, C. and S. Rajapakse, HIV, poverty and women. International Health (RSTMH), 2010. 2(1): p. 9-16.

10. Alvarez, M.J. and L. Garcia-Marques, Cognitive and contextual variables in sexual partner and relationship perception. Arch Sex Behav, 2011. 40(2): p. 407-17.

11. Murray, L., et al., The role of relationship intimacy in consistent condom use among female sex workers and their regular paying partners in the Dominican Republic. AIDS Behav, 2007. 11(3): p. 463-70.

12. Caldwell, K. and A. Mathews, The Role of Relationship Type, Risk Perception, and Condom Use in Middle Socioeconomic Status Black Women's HIV-prevention Strategies. Journal of black sexuality and relationships, 2015. 2(2): p. 91-120.

13. Fonner, V.A., et al., Do Sexual Partners Talk to Each Other About HIV? Exploring Factors Associated with HIV-Related Partner Communication Among Men and Women in Tanzania. AIDS and Behavior, 2020. 24(3): p. 891-902.

14. Kwok, Q., J. Chau, and E. Holroyd, Examining the relationships between condom use self-efficacy and condom use among mainland Chinese sex workers in Hong Kong. Japan Journal of Nursing Science, 2010. 28: p. 23-32.

15. Nesoff, E.D., K. Dunkle, and D. Lang, The Impact of Condom Use Negotiation Self-Efficacy and Partnership Patterns on Consistent Condom Use Among College-Educated Women. Health Educ 
Behav, 2016. 43(1): p. 61-7.

16. Bryan, A.E.B., et al., Condom-Insistence Conflict in Women's Alcohol-Involved Sexual Encounters with a New Male Partner. Psychology of women quarterly, 2017. 41(1): p. 100-113.

17. Sunmola, A., B. Olley, and G. Oso, Predictors of condom use among sexually active persons involved in compulsory national service in Ibadan, Nigeria. Health education research, 2007. 22: p. 459-72.

18. Devine-Wright, H., et al., Correlates of condom use and condom-use motivation among young South Africans. Journal of Applied Social Psychology, 2015. 45(12): p. 674-683.

19. Mpondo, F., et al., Self-determination and gender-power relations as predictors of condom use selfefficacy among South African women. Health Psychology Open, 2015. 2(2): p. 2055102915598676.

20. Bandura, A., Self-efficacy: Toward a unifying theory of behavioral change. Advances in Behaviour Research and Therapy, 1978. 1(4): p. 139-161.

21. Asante, K.O. and P.N. Doku, Cultural adaptation of the Condom Use Self Efficacy Scale (CUSES) in Ghana. BMC Public Health, 2010. 10(1): p. 227.

22. Brafford, L.J. and K.H. Beck, Development and validation of a condom self-efficacy scale for college students. J Am Coll Health, 1991. 39(5): p. 219-25.

23. Brien, T.M., et al., Dimensions of self-efficacy among three distinct groups of condom users. J Am Coll Health, 1994. 42(4): p. 167-74.

24. Coffman, D.L., et al., Effects of HealthWise South Africa on Condom Use Self-efficacy. Prevention Science, 2011. 12(2): p. 162-172.

25. Ajayi, A.I., K.O. Ismail, and W. Akpan, Factors associated with consistent condom use: a crosssectional survey of two Nigerian universities. BMC public health, 2019. 19(1): p. 1207.

26. Ajayi, A.I., et al., Low awareness and use of post-exposure prophylaxis among adolescents and young adults in South Africa: implications for the prevention of new HIV infections. African Journal of AIDS Research, 2020. 19(3): p. 242-248.

27. Ajayi, A.I., et al., Low awareness and use of pre-exposure prophylaxis among adolescents and young adults in high HIV and sexual violence prevalence settings. Medicine, 2019. 98(43).

28. Barkley Jr, T.W. and J.L. Burns, Factor analysis of the Condom Use Self-Efficacy Scale among multicultural college students. Health Education Research, 2000. 15(4): p. 485-489.

29. Mhlongo, S., et al., Factors associated with not testing for HIV and consistent condom use among men in Soweto, South Africa. PLoS One, 2013. 8(5): p. e62637.

30. Moyo, W., et al., Consistent condom use in South African youth's most recent sexual relationships. AIDS and Behavior, 2008. 12(3): p. 431-440.

31. Jama Shai, N., et al., Factors associated with consistent condom use among rural young women in South Africa. AIDS care, 2010. 22(11): p. 1379-1385.

32. Nyembezi, A., et al., Correlates of consistent condom use among recently initiated and traditionally circumcised men in the rural areas of the Eastern Cape Province, South Africa. BMC Public Health, 2014. 14(1): p. 668. 
33. Anyangu, A.S., Prevalence and factors influencing consistent condom use among sexually active young people attending a youth friendly centre in Kenya, 2008. East African journal of public health, 2010. 7(4).

34. Ajayi, A.I. and S.R. Okeke, Protective sexual behaviours among young adults in Nigeria: influence of family support and living with both parents. BMC Public Health, 2019. 19(1): p. 983. 